\title{
Fast Technique of Impulsive Noise Suppression in Color Images
}

\author{
Bogdan Smolka \\ Silesian University of Technology, Akademicka 16, 44-100 Gliwice, Poland \\ Bogdan.Smolka@polsl.pl
}

\begin{abstract}
In this work a novel technique of impulsive noise removal in color images is proposed. The new approach is based on the calculation of costs of digital paths which link the central pixel of the processing window with its boundary. The minimum value of these costs serves as a measure of pixel impulsiveness. The output of the proposed filtering technique is a weighted average of the central pixel and its robust estimate, calculated utilizing the measures of corruption assigned to pixels in the local neighborhood.

The experiments performed on a set of standard color images revealed a very high effectiveness of the new filtering design, comparable with the most efficient methods known from the literature. Additionally, the new filter is extremely fast and therefore it can be applied in real time image enhancement scenarios.
\end{abstract}

Keywords: impulsive noise, color image enhancement.

\section{Introduction}

Noise reduction, despite many years of investigations, remains an active research field, as the restoration and enhancement of noisy images is an indispensable step in most imaging pipelines. Quite often color images are corrupted by impulsive noise, caused by the instabilities in the image formation process, poor lighting conditions, electromagnetic interferences and aging of the storage material $^{(1,2)}$.

Numerous filters, which were elaborated to suppress the impulsive noise in color images, are based on order statistics $^{(3-5)}$. These methods rely on the ordering of color pixels of the processing window $W$, which are treated as vectors. For each pixel from $W$ the sum of distances to neighboring pixels is assigned, the cumulative distances are sorted and the ordered sequence of color pixels is utilized in the construction of various filtering designs. The popular Vector Median Filter (VMF) output is the first ordered pixel and it minimizes the sum of distances to other samples in the processing window. The $\alpha$-trimmed VMF averages the first $\alpha$ of the ordered pixels to enable the additional smoothing of Gaussian noise contaminating the color image.

The efficiency of these techniques is limited in the case of impulsive noise, as every image pixel is processed and mostly changed, which leads to the unnecessary filtering of uncorrupted pixels, resulting in the loss of image details. Therefore, the switching filters, which replace only the pixels detected as corrupted by the impulsive noise, offer much better denoising quality ${ }^{(6,7)}$.

The efficiency of the switching filters is strongly dependent on the accuracy of the impulse detection scheme and the proper adjustment of the threshold, which discriminates between the impulses and uncorrupted pixels. Therefore, the soft-switching filters, whose output is defined as a weighted average of the central pixel of the processing window and a robust pixel estimate like VMF, offer better image enhancement results. Their main advantage is that no thresholding is performed and their output is not so sensitive to the inaccurate estimation of the degree of pixel corruption ${ }^{(8,9)}$.

The paper is structured as follows. In Section 2 the design of the proposed filtering technique is described. In the next Section the results of experiments and the comparison with the state-of-the-art denoising methods are presented. Section 5 concludes the paper.

\section{Filter design}

Let $D$ be an nonempty set. We can assign distances to points in $D$, defining a real valued function on the Cartesian product $D \times D$. The function $\rho: D \times D \rightarrow \mathbb{R}$ is called a distance if it satisfies: $\rho(u, v) \geq 0$, with $\rho(u, v)=0$ when $u=v$, for $u, v \in$ $D \times D$. In digital image processing three basic distances are frequently used. If $u=\left(u_{1}, u_{2}\right)$ and $\mathrm{v}=\left(v_{1}, v_{2}\right)$ denote two image points, $\left(u, v \in \mathbb{Z}^{2}\right)$, then we can define the city-block distance 
$\mathrm{L}_{1}$, the Euclidean $\mathrm{L}_{2}$ and chessboard $\mathrm{L}_{\infty}$ distance:

$$
\begin{aligned}
& \rho_{1}(u, v)=\left|u_{1}-v_{1}\right|+\left|u_{2}-v_{2}\right|, \\
& \rho_{2}(u, v)=\left(\left(u_{1}-v_{1}\right)^{2}+\left(u_{2}-v_{2}\right)^{2}\right)^{1 / 2}, \\
& \rho_{\infty}(u, v)=\max \left\{\left|u_{1}-v_{1}\right|+\left|u_{2}-v_{2}\right|\right\} .
\end{aligned}
$$

Using the city-block and chessboard distances, we are able to define the two basic types of neighborhood: 4-neighborhood $\mathcal{N}_{4}(u)=\left\{v: \rho_{4}(u, v)=1\right\}$ and 8-neigborhood $\mathcal{N}_{8}(u)=\left\{v: \rho_{\infty}(u, v)=1\right\}$. In this paper we will assume that the image pixels are in 8-neighborhood relation and will consider digital paths defined as a sequence of points $\left(x_{0}\right.$, $\left.x_{1, \ldots,}, x_{\eta}\right)$, such that for $k \in\{1,2, \ldots, n\} x_{k-1} \neq x_{k}$.

Using the distances between neighboring points, which are called prime distances, we can define a distance between any pair of image pixels by following all admissible paths linking them and then taking the minimum of the total length over all possible routes. In this way, the distance between two points on the image domain is the length of the path for which the sum of prime distances between the path nodes is minimal. For the city-block distance, the admissible paths consist of horizontal and vertical moves, whereas for the chessboard distance also the diagonal moves are allowed. The prime distances of the two kinds of neighborhoods are assumed in this paper to be equal to $\rho=1$. The cost $C\left(q_{0}, q_{n}\right)$ of a path joining the image pixels $q_{0}, q_{1, \ldots, q_{\mathrm{n}} \text { will be defined as }}$

$$
C\left(x_{0}, x_{n}\right)=\sum_{k=1}^{n} \rho\left(x_{k-1}, x_{k}\right) .
$$

For gray scale images, the concept of the cost of digital paths can be extended, so that the differences in pixel intensities between neighboring pixels are taken into consideration. Then the cost of a path can be defined as

$$
C\left(x_{0}, x_{\eta}\right)=\sum_{k=1}^{n}\left(\left|I\left(x_{k-1}\right)-I\left(x_{k-1}\right)\right|^{\gamma}+\lambda\right) .
$$

The cost of a transition between two image pixels is thus a sum of absolute intensity differences encountered on a given path raised to the power of $\gamma$ and combined with the scaled topographic distance parameter $\lambda$. The minimal cost of a transition between two image points is called a geodesic distance.

For color images, denoting the image pixels as $\mathbf{x}_{k}$, where $k$ is an index indicating at the pixel position on the image domain, the cost of a path can be expressed as

$$
C\left(x_{0}, x_{\eta}\right)=\sum_{k=1}^{\eta}\left(\left\|\mathbf{x}_{k-1}-\mathbf{x}_{k}\right\|^{\gamma}+\lambda\right),
$$

where $\|\cdot\|$ denotes the Euclidean norm in RGB color space.

In the article ${ }^{10}$ the minimum cost of a path joining the boundary of a processing window with its center has been used as a measure of pixel distortion. The rationale behind such an approach is the fact that isolated pixels, which are introduced by the impulsive noise process, are not included in any low-cost path leading from the center to the border of a filtering window.

Figure 1 shows an exemplary $5 \times 5$ processing window, whose central pixel has the intensity 9 , which is outlying from other samples. The minimum cost of a path connecting this pixel with the window's border-shaded pixels-will serve as a measure of its outlyingness.

\begin{tabular}{|l|l|l|l|l|}
\hline 1 & 5 & 1 & 3 & 0 \\
\hline 3 & 2 & 2 & 1 & 5 \\
\hline 7 & 1 & 9 & 0 & 4 \\
\hline 3 & 3 & 2 & 1 & 3 \\
\hline 2 & 4 & 8 & 2 & 0 \\
\hline
\end{tabular}

Fig. 1. Exemplary processing window of size $5 \times 5$ with depicted gray scale intensities.

\begin{tabular}{|l|l|l|l|l|}
\hline 1 & & 1 & & 0 \\
\hline & 2 & 2 & 1 & \\
\hline 7 & 1 & 9 & 0 & 4 \\
\hline & 3 & 2 & 1 & \\
\hline 2 & & 8 & & 0 \\
\hline
\end{tabular}

Fig. 2. Digital paths of length 2 joining the border of the processing window with its center.

\begin{tabular}{|c|c|c|c|c|}
\hline $\begin{array}{c}i-2, \\
j-2\end{array}$ & & $\begin{array}{c}i-2, \\
j\end{array}$ & & $\begin{array}{c}i-2, \\
j+2\end{array}$ \\
\hline & $\begin{array}{c}i-1, \\
j-1\end{array}$ & $\begin{array}{c}i-1, \\
j\end{array}$ & $\begin{array}{c}i-1, \\
j+1\end{array}$ & \\
\hline $\begin{array}{c}i, \\
j-2\end{array}$ & $\begin{array}{c}i, \\
j-1\end{array}$ & $\begin{array}{c}i, \\
j\end{array}$ & $\begin{array}{c}i, \\
j+1\end{array}$ & $\begin{array}{c}i, \\
j+2\end{array}$ \\
\hline & $\begin{array}{c}i+1, \\
j-1\end{array}$ & $\begin{array}{c}i+1, \\
j\end{array}$ & $\begin{array}{c}i+1, \\
j+1\end{array}$ & \\
\hline $\begin{array}{c}i+2, \\
j-2\end{array}$ & & $\begin{array}{c}i+2, \\
j\end{array}$ & & $\begin{array}{c}i+2, \\
j+2\end{array}$ \\
\hline
\end{tabular}

Fig. 3. Notation used for the description of paths.

For the determination of the minimum cost of a path joining the window's center with its border a double scan algorithm can be employed ${ }^{(11,12)}$. This algorithm, in its simplified form, performs the running analysis of five 
pixels in a local moving window, which moves through the image in two scans.

To decrease the computational complexity, only the paths in horizontal, vertical and diagonal direction joining the border of the window with its center are considered in this paper. In the example shown in Fig. 1, the center of the window can be reached in two steps as shown in Fig. 2.

Using the notation presented in Fig. 3, and setting $\gamma=2$ and $\lambda=0$, the costs of 8 digital paths in horizontal, vertical and diagonal direction of length $n=2$ are:

$$
\begin{aligned}
& C_{1}=\frac{1}{n} \sum_{k=1}^{n}\|\mathbf{x}(i, j+k)-\mathbf{x}(i, j+k-1)\|^{2}, \\
& C_{2}=\frac{1}{n} \sum_{k=1}^{n}\|\mathbf{x}(i-k, j+k)-\mathbf{x}(i-k+1, j+k-1)\|^{2}, \\
& C_{3}=\frac{1}{n} \sum_{k=1}^{n}\|\mathbf{x}(i-k, j)-\mathbf{x}(i-k+1, j)\|^{2}, \\
& C_{4}=\frac{1}{n} \sum_{k=1}^{n}\|\mathbf{x}(i-k, j-k)-\mathbf{x}(i-k+1, j-k+1)\|^{2}, \\
& C_{5}=\frac{1}{n} \sum_{k=1}^{n}\|\mathbf{x}(i, j-k)-\mathbf{x}(i, j-k+1)\|^{2}, \\
& C_{6}=\frac{1}{n} \sum_{k=1}^{n}\|\mathbf{x}(i+k, j-k)-\mathbf{x}(i+k-1, j-k+1)\|^{2}, \\
& C_{7}=\frac{1}{n} \sum_{k=1}^{n}\|\mathbf{x}(i+k, j)-\mathbf{x}(i+k-1, j)\|^{2}, \\
& C_{8}=\frac{1}{n} \sum_{k=1}^{n}\|\mathbf{x}(i+k, j+k)-\mathbf{x}(i+k-1, j+k-1)\|^{2},
\end{aligned}
$$

where $C_{k}$ is the cost of a path in the direction $k$, where $k=1,2 \ldots, 7,8$, denotes the geographic directions (E,NE,..,S, $\mathrm{SE})$. The minimum cost $C=\min \left\{C_{k}\right\}$ will serve as a measure of pixel corruption.

The simplest scheme for the replacement of the noisy pixels would be to construct a switching filter based on the minimum connection cost, so that if it exceeds a specified threshold, then the pixel would be replaced by a robust estimate like VMF and otherwise this pixel would not be changed. However, such a design would be very sensitive to the thresholding parameter value and therefore a soft-switching technique based on the idea presented in paper $^{(10)}$ was implemented.

In the first step, a local weighted average $\mathbf{y}_{i}$ of pixels in a $3 \times 3$ window $W$ centered at position $i$ is determined

$$
\mathbf{y}_{i}=\frac{1}{S} \sum_{k=1}^{9} \mathbf{x}_{k} \cdot \exp \left(-\frac{C_{k}^{2}}{h_{1}^{2}}\right), \quad S=\sum_{k=1}^{9} \exp \left(-\frac{C_{k}^{2}}{h_{1}^{2}}\right),
$$

where $C_{k}$ are the minimum costs of paths calculated for pixels $\mathbf{x}_{k} \in \mathrm{W}$ according to Eq. (5). In this way $\mathbf{y}_{i}$ is the weighted average of the pixels in the local neighborhood and the weights are small for the noise affected pixels. The estimate of the uncorrupted color pixel is then used in the second step, which provides the final filter output $\mathbf{z}_{i}$

$$
\mathbf{z}_{i}=w_{i} \cdot \mathbf{x}_{i}+\left(1-w_{i}\right) \cdot \mathbf{y}_{i}, w_{i}=\exp \left(-\frac{C_{i}}{h_{2}^{2}}\right) .
$$

The output pixel is an average of the pixel $\mathbf{x}_{i}$ of the noisy image and its estimate $\mathbf{y}_{i}$. The weights in (6) and (7) are determined by the minimum path costs and are controlled by parameters $h_{1}$ and $h_{2}$ which have to be tuned to obtained proper denoising results.

\section{Experiments}

The efficiency of the proposed technique of impulsive noise removal was evaluated on a set of color test images depicted in Fig. 4. These images were corrupted by random valued impulsive noise, so that each image pixel was contaminated with probability $p$. The RGB channels of corrupted pixels were replaced by random values from the range $[0,255]$ drawn from a uniform distribution.

For the assessment of the restoration quality, the PSNR and MAE quality measure were used

$$
\begin{gathered}
\mathrm{PSNR}=10 \log _{10}\left\{\frac{255^{2}}{\mathrm{MSE}}\right\}, \mathrm{MSE}=\frac{1}{3 \mathrm{Q}} \sum_{i=1}^{\mathrm{Q}} \sum_{k=1}^{3}\left(x_{i k}-z_{i k}\right)^{2}, \\
\operatorname{MAE}=\frac{1}{3 \mathrm{Q}} \sum_{i=1}^{\mathrm{Q}} \sum_{k=1}^{3}\left|x_{i k}-z_{i k}\right|,
\end{gathered}
$$

where $x_{i k}$ and $z_{i k}$ stand for the noise-free and restored pixel channels and $Q$ denotes the number of image pixels.

Figure 5 depicts the influence of the parameters $h_{1}$ and $h_{2}$ on the PSNR values. As can be observed, the best achievable results are only slightly dependent on the image contamination intensity and its structure. Similar conclusions can be drawn from the dependence of MAE quality measures on the two parameters. Therefore, a default setting $h_{1}=h_{2}=30$ and the $5 \times 5$ window was adopted for the comparison of the new filter with techniques known from the literature.

For the evaluation of the new filter's performance, it has been compared with a set of efficient methods ${ }^{(6)}$. The following filters were taken for comparisons: Soft Switching Filter ${ }^{(10)}$ (SSF), Fast Averaging Peer Group Filter $^{(7)}$ (FAPGF), Sigma Vector Median Filter (SVMF), Sigma Directional-Distance Filter (SDDF), Peer Group Filter (PGF), Fast Modifed Vector Median Filter (FMVMF), 
Adaptive Vector Median Filter (AVMF), Adaptive Center Weighed Vector Median Filter (ACWVMF), Adaptive Center Weighed Directional Distance Filter (ACWDDF) and Fast Peer Group Filter (FPGF).

The filtering results presented in Fig. 6 and summarized in Tab. 1 in terms of the PSNR and MAE quality measures $(\mathrm{QM})$, prove the very high efficiency of the new filtering structure. Although, the results are slightly worse than those achieved using the $\operatorname{SSF}^{(10)}$, the loss of efficiency is compensated by much lower computational complexity. For the estimation of pixel impulsiveness, only 16 distances between pixels has to be computed, as only horizontal, vertical and diagonal paths are considered. The calculation of weights, needed for the determination of the final output, do not significantly increase the computational burden. Furthermore, the new filter performance is comparable with the newly introduced $\mathrm{FAPG}^{(7)}$, which belongs to the most efficient denoising frameworks.

\section{Conclusions}

In the paper a novel filtering design intended for the suppression of impulsive noise in color images has been presented. The proposed method is based on the cost of horizontal, vertical and diagonal digital paths which join the central pixel of a processing window with its border. The central pixel is assigned the minimum cost, which serves as a measure of its corruption. This feature is used in the construction of a soft switching filter whose output is a weighted average of the processed sample and its robust estimate. The filter is controlled by two parameters, which proved to be not dependent on the image structure and noise intensity level.

Besides its high efficiency, comparable with best existing techniques, the new technique is very fast, as for the estimation of pixel corruption measure only 16 distances between pixels in the filtering window have to be computed and the robust estimate needed for the switching weight is calculated in a $3 \times 3$ window. The structure of the filter is quite simple and can be easily parallelized, so that it can be applied in various real time imaging tasks.

\section{Acknowledgment}

This work has received funding from statutory funds (BK/213/RAU1/2016) of the Institute of Automatic Control, Silesian University of Technology, Poland.

\section{References}

(1) K.N. Plataniotis, A.N. Venetsanopoulos, "Color Image Processing and Applications", Springer, 2000

(2) C.G. Boncelet, "Image noise models", in: Handbook of Image and Video Processing, Communications, Networking and Multimedia, pp. 397-410. Academic Press, 2005

(3) R. Lukac, B. Smolka, K. Martin, K.N. Plataniotis, A.N. Venetsanopoulos, "Vector filtering for color imaging", IEEE Signal Processing Magazine, Vol. 22, No. 1, pp. 74-86, 2005

(4) B. Smolka, K.N. Plataniotis, A.N. Venetsanopoulos, "Nonlinear techniques for color image processing", in: Nonlinear Signal and Image Processing, pp. 445-505, CRC Press, 2004

(5) B. Smolka, A.N. Venetsanopoulos, "Noise reduction and edge detection in color images", in: Color Image Processing: Methods and Applications, pp. 75-100, CRC Press, 2006

(6) M. Celebi, H. Kingravi, Y. Aslandogan, "Nonlinear vector filtering for impulsive noise removal from color images", Journal of Electronic Imaging, Vol. 16, No. 3, 033008, 2007

(7) L. Malinski and B. Smolka, "Fast averaging peer group filter for the impulsive noise removal in color images", Journal of Real-Time Image Processing, Vol. 11, No. 3, pp. 427-444, 2016

(8) S. Morillas, S. Schulte, T. Melange, E. Kerre, V. Gregori, "A soft switching approach to improve visual quality of colour image smoothing filters," Lecture Notes in Computer Science, Vol. 4678, pp. 254-261, 2007

(9) B. Smolka, "Soft switching technique for impulsive noise removal in color images", Proceedings of Fifth International Conference on Computational Intelligence, Communication Systems and Networks (CICSyN), pp. 222-227, 2013

(10)B. Smolka, B. Cyganek, "Impulsive noise suppression in color images based on the geodesic digital paths", Proc. SPIE, Vol. 9400, pp. 94000R-94000R-12, 2015

(11)P.J. Toivanen, "New geodesic distance transforms for gray-scale images", Pattern Recognition Letters, Vol. 17, No. 5, pp. 437 - 450, 1996

(12) G. Borgefors, "Distance transformations in digital images", Computer Vision, Graphics, and Image Processing, Vol. 34, pp. 344-371, 1986 
Table 1: Filtering efficiency of the NEW technique compared with competitive denoising methods.

\begin{tabular}{|c|c|c|c|c|c|c|c|c|c|c|c|c|}
\hline QM & $p$ & SSF & NEW & FAPGF & SVMF & SDDF & PGF & FMVMF & AVMF & ACWVMF & ACWDDF & FPGF \\
\hline \multicolumn{13}{|c|}{ CAPS } \\
\hline \multirow[t]{3}{*}{ PNSR } & 0.1 & 40.47 & 39.77 & 40.10 & 37.27 & 33.18 & 39.72 & 38.58 & 31.94 & 39.17 & 36.57 & 38.47 \\
\hline & 0.2 & 37.68 & 36.94 & 36.75 & 30.25 & 26.75 & 34.40 & 34.96 & 28.51 & 32.36 & 31.69 & 34.50 \\
\hline & 0.3 & 35.31 & 34.65 & 34.11 & 24.34 & 22.25 & 29.65 & 30.06 & 25.67 & 26.66 & 26.77 & 29.83 \\
\hline \multirow[t]{3}{*}{ MAE } & 0.1 & 0.50 & 0.60 & 0.39 & 0.64 & 1.05 & 0.38 & 0.42 & 0.97 & 0.38 & 0.57 & 0.45 \\
\hline & 0.2 & 0.89 & 1.06 & 0.80 & 1.29 & 2.23 & 0.88 & 0.84 & 2.06 & 1.01 & 1.18 & 0.96 \\
\hline & 0.3 & 1.33 & 1.52 & 1.32 & 3.26 & 4.79 & 1.74 & 1.64 & 3.53 & 2.38 & 2.39 & 1.83 \\
\hline \multicolumn{13}{|c|}{ FLOWERS } \\
\hline \multirow[t]{3}{*}{ PNSR } & 0.1 & 38.41 & 37.64 & 38.22 & 36.07 & 33.46 & 37.86 & 36.39 & 30.88 & 36.93 & 35.87 & 36.26 \\
\hline & 0.2 & 35.49 & 34.93 & 35.05 & 30.35 & 27.69 & 33.19 & 33.40 & 27.54 & 31.91 & 31.69 & 32.87 \\
\hline & 0.3 & 33.85 & 33.07 & 32.67 & 25.08 & 23.44 & 29.43 & 29.64 & 25.02 & 27.31 & 27.59 & 29.42 \\
\hline \multirow[t]{3}{*}{ MAE } & 0.1 & 0.65 & 0.79 & 0.49 & 0.67 & 1.02 & 0.49 & 0.56 & 1.20 & 0.53 & 0.71 & 0.64 \\
\hline & 0.2 & 1.20 & 1.40 & 1.02 & 1.47 & 2.15 & 1.19 & 1.13 & 2.57 & 1.30 & 1.43 & 1.35 \\
\hline & 0.3 & 1.70 & 2.01 & 1.68 & 3.30 & 4.35 & 2.20 & 2.07 & 4.28 & 2.64 & 2.62 & 2.41 \\
\hline \multicolumn{13}{|c|}{ RAFTING } \\
\hline \multirow[t]{3}{*}{ PNSR } & 0.1 & 32.62 & 31.84 & 31.66 & 31.22 & 30.23 & 32.92 & 30.36 & 29.77 & 31.95 & 32.01 & 30.76 \\
\hline & 0.2 & 30.26 & 29.72 & 29.72 & 26.99 & 25.50 & 29.53 & 28.50 & 26.68 & 28.33 & 28.66 & 28.48 \\
\hline & 0.3 & 28.24 & 28.54 & 28.15 & 22.61 & 21.59 & 26.43 & 25.96 & 24.14 & 24.50 & 25.04 & 25.88 \\
\hline \multirow[t]{3}{*}{ MAE } & 0.1 & 1.49 & 1.76 & 1.29 & 1.60 & 2.03 & 1.06 & 1.59 & 1.55 & 1.21 & 1.52 & 1.61 \\
\hline & 0.2 & 2.52 & 2.97 & 2.25 & 2.78 & 3.57 & 2.19 & 2.55 & 3.14 & 2.44 & 2.66 & 2.80 \\
\hline & 0.3 & 3.54 & 3.54 & 3.32 & 5.40 & 6.48 & 3.76 & 4.03 & 5.18 & 4.48 & 4.43 & 4.48 \\
\hline \multicolumn{13}{|c|}{ AIRPLANE } \\
\hline \multirow[t]{3}{*}{ PNSR } & 0.1 & 35.81 & 35.81 & 34.42 & 32.66 & 31.34 & 35.03 & 32.18 & 31.79 & 33.47 & 34.05 & 32.75 \\
\hline & 0.2 & 32.81 & 32.81 & 32.12 & 25.40 & 25.30 & 30.13 & 29.00 & 28.01 & 27.30 & 29.25 & 29.24 \\
\hline & 0.3 & 31.06 & 31.06 & 30.07 & 19.67 & 20.65 & 24.67 & 24.00 & 23.49 & 21.65 & 24.05 & 24.06 \\
\hline \multirow[t]{3}{*}{ MAE } & 0.1 & 0.76 & 0.76 & 0.70 & 0.96 & 1.29 & 0.59 & 0.91 & 0.92 & 0.70 & 0.84 & 0.90 \\
\hline & 0.2 & 1.35 & 1.35 & 1.26 & 2.34 & 2.71 & 1.38 & 1.65 & 1.98 & 1.86 & 1.67 & 1.74 \\
\hline & 0.3 & 1.93 & 1.93 & 1.97 & 6.41 & 5.95 & 3.25 & 3.63 & 4.18 & 4.89 & 3.54 & 3.85 \\
\hline
\end{tabular}

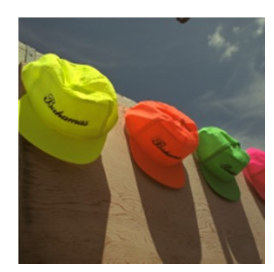

CAPS

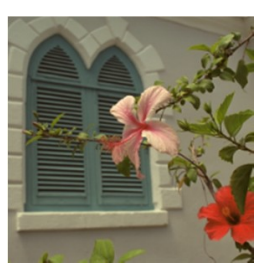

FLOWERS

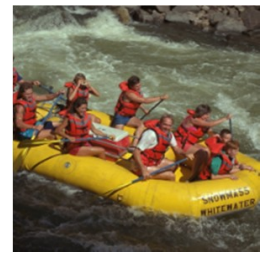

RAFTING

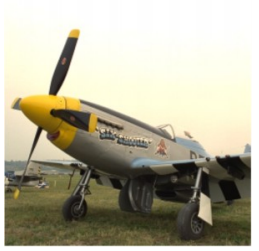

AIRPLANE

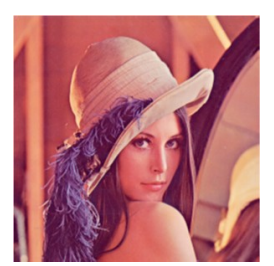

LENA

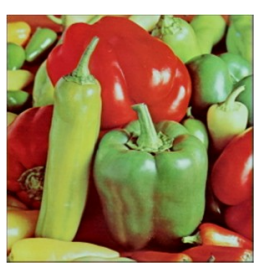

PEPPERS

Fig. 4. Color test images. 

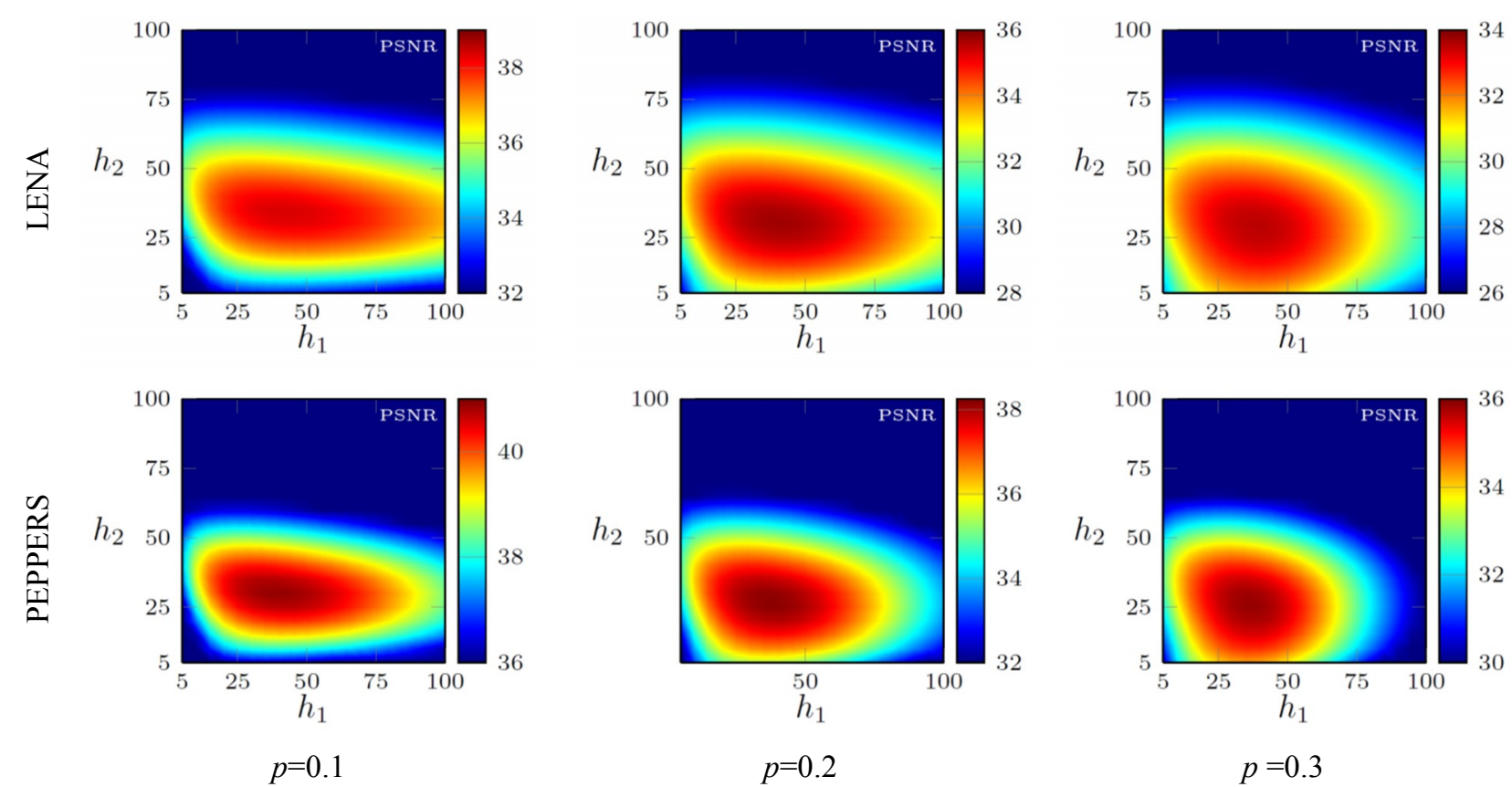

Fig. 5. Dependence of PSNR on the parameters $h_{1}$ and $h_{2}$ for the LENA and PEPPERS test images.

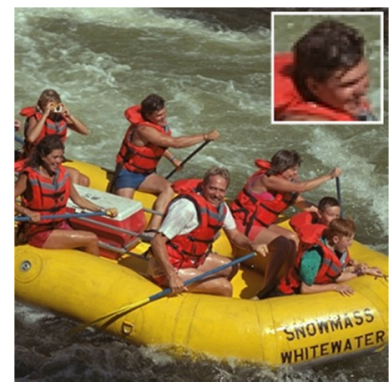

Test image

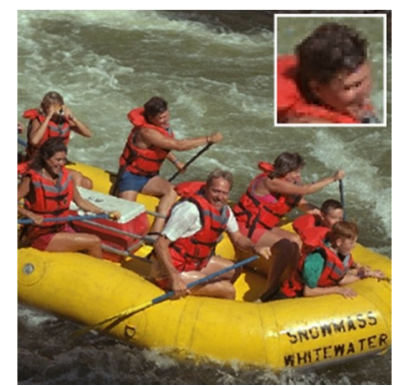

FAPGF

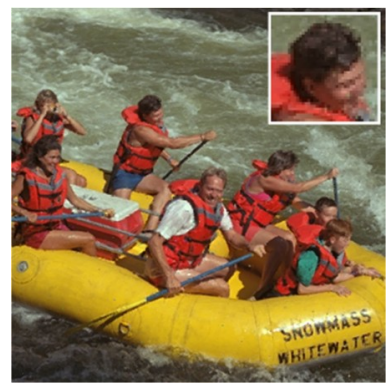

FMVMF

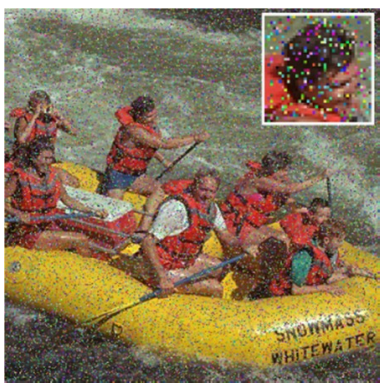

Noisy, $p=0.2$

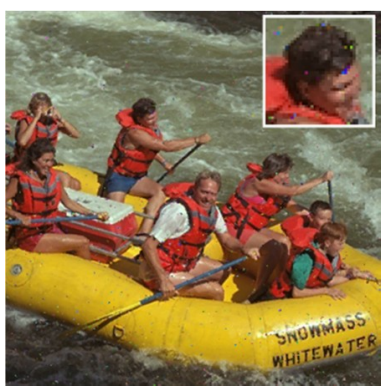

SVMF

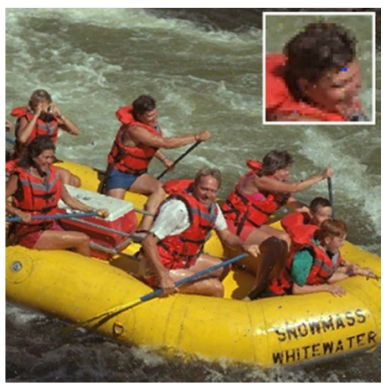

ACWVMF

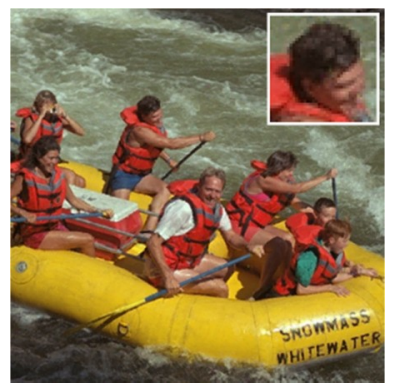

NEW

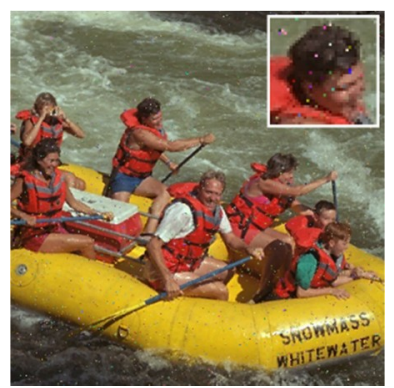

SDDF

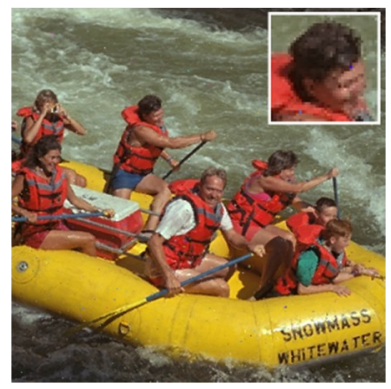

ACWDDF

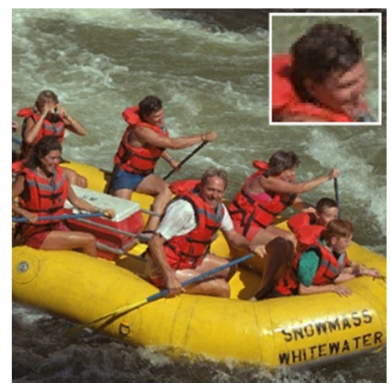

SSF

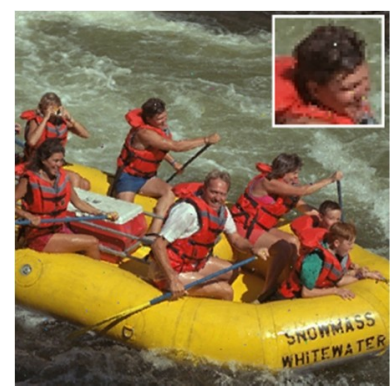

PGF

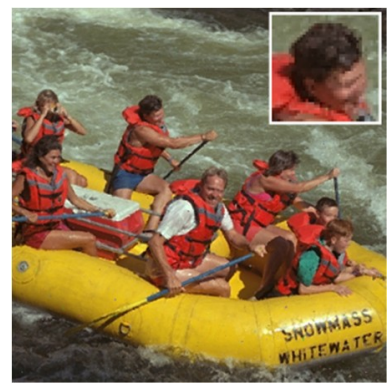

FPGF

Fig. 6. Filtering efficiency of the NEW filter compared with the competitive methods. 